\title{
All in the mind? Pain, placebo effect, and ergogenic effect of caffeine in sports performance
}

This article was published in the following Dove Press journal:

Open Access Journal of Sports Medicine

24 June 2010

Number of times this article has been viewed

\section{Christopher J Beedie \\ Department of Sports Science, Tourism and Leisure, Canterbury Christ Church, University, Canterbury, UK}

Correspondence: Christopher J Beedie Department of Sports Science, Tourism and Leisure, Canterbury Christ Church University, Canterbury, UK

Tel +440I2 27767700

Email christopher.beedie@canterbury. ac.uk

\begin{abstract}
The ergogenic effects of caffeine on performance are well documented. These effects are more evident in endurance and short-duration, sustained-effort events than in interactive or stop-go sports. Experimentally-induced placebo effects of caffeine on sports performance have also been observed in a number of recent studies. In the present paper it is argued that, given the nature of the sports in which caffeine effects are observed, the well documented hypoalgesic effects of caffeine, and the fact that pain is highly placebo-responsive, a reduction in perceived pain might be the common factor in both the biologic and placebo ergogenic effects of caffeine on sports performance. This idea is supported by evidence from medicine that suggests placebo effects are often associated with mechanisms similar or identical to those of the substance the subject believes they have ingested. Research findings from both biomedicine and sports medicine that attest to the interaction of biologic and psychologic factors in caffeine and pain responses are briefly reviewed. In conclusion, it is recommended that researchers investigate the pain hypothesis. Furthermore, researchers should consider psychosocial factors that might modulate the pain response as variables of interest in future caffeine and performance research.
\end{abstract}

Keywords: caffeine hypoalgesia, nocebo effects, research methods

\section{Introduction}

The ergogenic effects of caffeine on sports performance are well documented. ${ }^{1}$ Although these effects have been observed in a wide variety of sports, they are generally more evident in endurance and sustained high-intensity closed skill sports than in interactive and stop-go sports. ${ }^{2}$ The ergogenic effects of caffeine can be obtained at doses at or below the daily intake of normal populations, ${ }^{3}$ and below levels representing health risks. Caffeine is not currently proscribed by the World Anti-Doping Agency. Several mechanisms for the ergogenic effects of caffeine have been proposed, including enhanced fat oxidation, sympathetic nervous system enhancement, reduction in central fatigue, attenuation of neuromuscular conduction block, and potentiation of muscular force output for given input. ${ }^{4}$ These mechanisms are not consistently supported by research evidence, eg, Graham et al concluded that there is very little evidence to support the fat oxidation theory, ${ }^{1}$ whilst Tarnopolsky suggested there is no evidence for caffeine-induced changes in peripheral nerve conduction or neuromuscular transmission. ${ }^{4}$ The lack of consistent support for any one physiologic mechanism, and the fact that an increasing number of studies in sport and elsewhere have demonstrated significant and substantial placebo effects of caffeine, ${ }^{5-10}$ suggests that psychologic factors might play a significant role in caffeine effects. 
Perhaps the obvious psychologic factor to consider is pain. The proposal that caffeine ingestion reduces pain in performance is supported by both experimental findings and the anecdotal reports of athletes. ${ }^{5,11}$ Furthermore, caffeine-induced pain reduction could explain, in part, both biologic and placebo effects of caffeine on performance. Such a pain hypothesis is supported by a number of factors. Firstly, caffeine has well documented hypoalgesic or painreducing properties, ${ }^{12}$ secondly, pain is a limiting factor on performance, ${ }^{13}$ thirdly, pain is highly placebo responsive, ${ }^{14}$ and lastly, the sports in which ergogenic effects of caffeine have been observed tend to be those in which athletes experience substantial levels of sustained pain without the relief of regular disruption of activity associated with stop-go or interactive sports. Given that pain is also highly socially modifiable and context-dependent, ${ }^{14}$ a pain hypothesis might explain the interindividual variability in caffeine response observed in sport research. ${ }^{15}$ The present paper aims to examine the potential influence of psychosocial factors in the ergogenic effect of caffeine in the context of caffeine-induced pain reduction. The article will review selected findings demonstrating placebo effects of caffeine on sports performance, hypoalgesic effects of caffeine on sports performance, and placebo effects on pain in experimental and clinical medicine. It will also briefly discuss implications for research.

\section{Placebo effects of caffeine on sports performance}

Placebo effects of caffeine on sports performance have been reported in three recent experimental studies. Beedie et al examined the possibility of a dose-response relationship to placebos presented as "zero", "low", and "high" dose caffeine among seven well-trained competitive cyclists. ${ }^{5}$ Measures were power, heart rate, oxygen uptake, and blood lactate. Following habituation and baseline trials, subjects were informed that, over three experimental trials, they would receive a placebo, or $4.5 \mathrm{mg} / \mathrm{kg}$ or $9.0 \mathrm{mg} / \mathrm{kg}$ caffeine double-blind and randomly assigned. However, a biologically inert placebo was administered in all experimental conditions. Postexperimental baseline trials were also conducted. A dose-response relationship was evident in experimental trials, with subjects producing $1.4 \%$ (range, $-4.6 \%-1.9 \%$ ) less power than baseline when they believed they had ingested a placebo, $1.3 \%(-1.4 \%-4.1 \%)$ more power than at baseline when they believed they had ingested $4.5 \mathrm{mg} / \mathrm{kg}$ caffeine, and $3.1 \%(0.4 \%-6.7 \%)$ more power than at baseline when they believed they had ingested $9.0 \mathrm{mg} / \mathrm{kg}$ caffeine. Of further interest was the fact that no substantial differences in any measured physiologic variables between baseline and experimental conditions were observed. Follow-up interviews with each subject indicated that five subjects believed that they had experienced a placebo effect, proposing mechanisms such as pain reduction, fatigue resistance, changes in strategy, and reduced arousal.

Pollo et al investigated the effects of a caffeine placebo on quadriceps muscle performance and perceived fatigue. ${ }^{6}$ Forty-four recreationally active males were divided into four groups, ie, two control and two placebo $(\mathrm{n}=11$ each). In the first experiment, a placebo was deceptively administered, with the suggestion that it was a high dose of caffeine. This resulted in a significant increase in mean muscle work $(11.8 \%-16.1 \%, P<0.01)$ but no perceived decrease in muscle fatigue $(P>0.05)$. In the second experiment, placebo caffeine administration was accompanied by a conditioning procedure whereby the weight to be lifted was surreptitiously reduced. The load was then restored to the original weight and placebo caffeine administered again. Compared with the first experiment, the placebo effect was larger, with a significant increase in muscle work $(22.1 \%-23.5 \%, P<0.01)$ and a decrease in perceived muscle fatigue $(-7.8-10.1, P<0.01)$. The authors suggested that their findings indicated a central mechanism of topdown modulation of the global performance of muscles by placebo and underscore the role of learning in the placebo response.

Foad et al used the balanced placebo design with 14 well-trained competitive cyclists in a study examining the effects of caffeine and placebo on $40 \mathrm{~km}$ laboratory cycling performance. ${ }^{7}$ Subjects performed two $40 \mathrm{~km}$ time trials in each of four experimental conditions (informed caffeine/received caffeine, informed no-treatment/received caffeine, informed caffeine/received placebo, and informed no-treatment/received no treatment). Measures were power, oxygen uptake, blood lactate, and heart rate. The authors reported a very likely beneficial main effect on mean power of receiving caffeine $(3.5 \% \pm 2.0 \%)$, and a possibly beneficial main effect of being informed of caffeine $(0.7 \% \pm 1.4 \%)$. A substantial interaction between belief and pharmacology $(2.6 \% \pm 3.3 \%)$ indicated that caffeine exerted a greater effect on performance when subjects were informed that they had not ingested it, while belief exerted a greater influence on performance in the absence of caffeine. A possibly harmful negative placebo (nocebo) effect relative to baseline was present when subjects were correctly informed that they had ingested no caffeine $(-1.9 \% \pm 2.2 \%)$. No substantial changes relative to baseline were observed in mean heart rate, although clear and substantial increases in blood lactate were 
evident following the receipt of caffeine. The within-subject $\mathrm{CV}$ for power in deceptive conditions at $2.8 \%$ was 1.7 times larger than the $\mathrm{CV}$ when subjects were truthfully informed that they were receiving caffeine, indicating the possibility of some disparity between internal sensations and instructions amongst some subjects. The authors suggested that their data supported the ergogenic efficacy of caffeine, but noted that in the absence of caffeine, the negative effect on performance of negative expectation was somewhat more substantial than the positive effect of positive expectation (a finding that could inflate effect sizes in placebo-controlled studies).

Evidence for placebo effects associated with caffeine has also been provided elsewhere. ${ }^{8-10}$ It is evident from these findings that whilst caffeine ingestion often exerts an influence on behavior, so too can beliefs about caffeine, and about whether or not caffeine has been ingested. These findings beg several questions as to the likely ergogenic mechanisms of caffeine and suggest a significant psychologic contribution.

\section{Hypoalgesic effects of caffeine in sports performance}

As suggested above, perhaps the most likely psychologic contribution would be pain reduction. There is a large body of evidence attesting to the hypoalgesic properties of caffeine in both medicine ${ }^{16}$ and physical activity. ${ }^{5,12,17,18}$ Consistent with this are data demonstrating that subjects' ratings of perceived exertion are lower when given caffeine. ${ }^{19}$ Pain is a highly complex biopsychologic phenomenon, and even a brief overview of theories and potential mechanisms is well beyond the scope of this paper. It is reasonable to suggest that whilst pain is certainly not a necessary condition of sports performance, athletes in endurance and sustained shortduration sports routinely experience pain in competition. Many will experience pain with little respite for a substantial part, if not for the entirety, of their event (as stated above, it is in such sports that caffeine's ergogenic efficacy has been most widely demonstrated, lending further weight to the pain hypothesis). Gliottoni et al state that there is an expanding body of evidence that acute exercise is a natural stimulus that might transiently, safely, and reliably produce muscle pain, ${ }^{12}$ whilst Cook et al suggest that not only are descriptors used to describe pain during exercise similar to those that have been used to characterize clinical pain conditions, ${ }^{20}$ but that pain ratings during exercise as measured by the short form of the McGill Pain Questionnaire are nearly one standard deviation above the mean scores associated with other laboratory methods of inducing pain. ${ }^{21}$ Research has suggested that ingestion of caffeine significantly reduces the pain response during performance. ${ }^{12,17,18,22,23}$ O'Connor et al reported that caffeine ingestion has a dose-response effect on reducing leg muscle pain during exercise. ${ }^{22}$ Motl et al reported that leg muscle pain ratings were significantly and moderately reduced after a high dose $(10 \mathrm{mg} / \mathrm{kg}$ body weight) of caffeine. ${ }^{18}$ Maridakis et al reported that caffeine produced a large and statistically significant hypoalgesia effect during maximal quadriceps contraction. ${ }^{23}$ Gliottoni and Motl reported that caffeine administration resulted in a large reduction in leg muscle pain intensity ratings. ${ }^{17}$ Gliottoni et al reported that caffeine ingestion is associated with a moderate hypoalgesic effect during high-intensity cycling. ${ }^{12}$ Theoretically, any intervention that reduces perceived exertion or pain should also increase the perceived headroom for effort, and therefore has the potential to enhance performance. A hypoalgesic effect driven by caffeine could be direct, ie, the action of caffeine attenuates the perception of pain. Indirectly, caffeine actions could influence other biologic processes that attenuate the pain response. The effect could also result from both direct and indirect processes simultaneously. Whichever way, and significantly for the present discussion, the large body of research in medicine and psychology demonstrating substantial placebo effects on pain could shed some light on the placebo effects related to caffeine and sports performance.

\section{Psychosocial modulation of pain}

Pain is highly susceptible to social and psychologic modulation, ${ }^{14}$ and perhaps because of this, over the last few years, pain has become one of the most fruitful areas of research into mind/body interaction. In short, evidence from such research demonstrates that expectation of pain relief can modify the subsequent effectiveness of administered substances, be they active analgesics, such as morphine, or inactive placebos. These effects can be both hypoalgesic and hyperalgesic. Several complex designs have been used to elucidate this phenomenon, ranging from covert manipulation of experimental pain stimuli, to direct comparison of the effects of the hidden/deceptive administration of biologically active treatments with the overt administration of biologically inactive substances. Studies using such designs are informative in demonstrating the degree to which pain can be modified by psychosocial processes, such as conditioning and expectation. On that basis, they are of interest to those investigating or using interventions in which pain reduction might be a factor in their efficacy. Some illustrative examples of such studies are briefly described below. 
Montgomery and Kirsch exposed subjects to experimental pain at baseline, and in subsequent trials surreptitiously reduced the pain stimulus whilst a placebo analgesic cream was administered (the latter process designed to lead subjects to believe that the cream had reduced the pain). ${ }^{24}$ Subjects were then split into two groups, the first was correctly informed about the deception, and the second was not informed. On re-exposure to the pain at baseline level, subjects who had been correctly informed of the deception experienced no pain relief when the placebo analgesia cream was applied, whilst those in the second group reported substantially lower pain. The authors also assessed subjects' expectation of analgesia, and reported that this accounted for $49 \%$ of the observed effects. The authors concluded that an analgesic response can be conditioned, but that the conditioned response might be either reversed or suppressed by correct information or negative expectation respectively.

Levine et al administered active painkillers covertly and placebo painkillers openly to two groups of subjects following dental surgery. ${ }^{25,26}$ They reported that the overt injection of a saline placebo described as morphine was as effective as a covert injection of morphine. Similarly, Benedetti et al compared the open administration of five different painkillers with the hidden and automated administration of the same drugs. ${ }^{27-29}$ The authors reported that in hidden administration conditions the time taken for postoperative pain to diminish by $50 \%$ was greatly increased for all drugs compared with open administration. These findings suggest that anticipation of analgesia is a factor in perceived analgesia.

Pollo et al treated postoperative patients with a painkiller on request for three consecutive days, as well as with a saline placebo (patients were given the intravenous saline as a background infusion in addition to the routine analgesic treatment). ${ }^{30}$ Subjects were divided into three groups, the first being told nothing about the saline, the second that they had a 50:50 chance of receiving the painkiller or a placebo, and the third that the saline solution was a potent painkiller (these three conditions forming, respectively, natural history, double-blind, and deceptive administration groups). The authors reported that, compared with the natural history group, a $20 \%$ decrease in requests for analgesia was observed in the double-blind group, and a $34 \%$ decrease in requests was observed in the deceptive administration group. The authors concluded that instructions that induce a certain expectation of analgesia induce greater placebo analgesia than those that induce uncertain expectation, a finding subsequently supported in a meta-analysis of 14

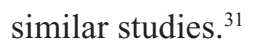

In an interesting twist on their open-versus-hidden administration design above, Benedetti et al used openversus-hidden interruption of morphine treatment in postoperative patients..$^{28,29}$ The authors reported that patients who were aware that their treatment had been discontinued were more likely to request further morphine than those who were unaware that their treatment had been interrupted. Similarly, Benedetti et al demonstrated pain increases associated with the administration of a placebo expected to increase pain in both clinical ${ }^{32}$ and experimental patients. ${ }^{33}$ The authors concluded that, in the same way that positive expectations of pain relief might induce or enhance actual pain relief, negative expectations might have the opposite effect.

\section{Evidence for biologic mechanisms of placebo and nocebo effects on pain}

Whilst early research into the psychosocial modulation of pain tended to focus on demonstrating the effect and speculating as to mechanisms, recent research has gone beyond this to investigating mechanisms in real time using technology such as positron emission tomography and magnetic resonance imaging. A classic experimental model is the administration of an agent known to antagonize the pathway that an administered placebo analgesic purports to mimic. For example, naloxone antagonizes the action of opiates such as morphine. Therefore, naloxone would also be expected to antagonize placebo analgesia if the same mechanisms as for morphine were responsible. In the first study of its kind, Levine et al demonstrated that naloxone did in fact disrupt placebo analgesia, concluding, logically, that the endogenous opioids are involved in the placebo response. ${ }^{34}$ These findings were replicated several times in both experimental and clinical settings. At the University of Turin, Benedetti et al further demonstrated that as well as the opioids, cholecystokinin, a neurotransmitter with an antiopioid action, plays a role in both placebo analgesia ${ }^{35}$ and nocebo hyperalgesia. ${ }^{32}$ Subsequent research has demonstrated, for example, placebo-induced activation of brain areas involved in the pain response, ${ }^{36}$ placebo-induced deactivation of brain areas involved in pain processing, ${ }^{37}$ and correlations between the magnitude of placebo analgesia and dopamine activity. ${ }^{38}$

In a study of the placebo analgesia mechanism related to sports performance, Benedetti et al investigated the placebo analgesic effects of morphine on a pain endurance test. ${ }^{39}$ Subjects had a tourniquet wrapped around their forearm and were required to squeeze a hand spring exerciser repeatedly until they could no longer continue. During precompetition 
training, two "teams", A and B, received no pharmacologic substance whilst teams $C$ and D were trained with morphine. During competition, team A received no treatment while teams $\mathrm{B}$ and $\mathrm{C}$ were given placebo morphine one hour before competition. Team $\mathrm{D}$ also received what they believed was morphine, but they actually received naloxone (which would be expected to antagonize the opioid pathways). As hypothesized, the largest placebo effect on pain tolerance was observed in team $\mathrm{C}$ who received both the morphine preconditioning in the "training" trials and also believed they had ingested morphine in the competition trials. In team D, who had received morphine in preconditioning trials, naloxone negated the morphine preconditioning effects. These findings suggest conditioned activation of endogenous opioids after placebo administration, although a correlation between morphine and placebo suggests the possible contribution of nonopioid mechanisms. It is noteworthy that the placebo analgesic responses were obtained after only two morphine administrations separated by as much as a week. These long time intervals suggest that pharmacologic conditioning procedures have long-lasting effects (with implications for the use of proscribed drugs in training and competition).

Collectively, the findings above and many others suggest not only that placebo effects have biologic mechanisms, but that these mechanisms may be similar or identical to those of the drug the placebo mimics. Given this, and given the reduced ratings of perceived exertion/pain observed in both caffeine and placebo caffeine research, it is reasonable to speculate that pain reduction might be one mechanism by which both the biologic and placebo effects of caffeine on sports performance operate.

\section{Implications}

It is proposed above that pain might be a factor in the observed ergogenic effect of both caffeine and placebo caffeine on sports performance. This proposal was supported by a brief review of research that has demonstrated the placebo effects of caffeine on sports performance, the hypoalgesic effects of caffeine in exercise, and the psychosocial modulation of pain responses. It is suggested that if pain reduction is a key factor in the ergogenic effect of caffeine, the findings of the latter body of research will be instrumental in elucidating mechanisms and explaining interindividual variability to response to caffeine. Specifically, these findings suggest that: an analgesic response can be conditioned and that the conditioned response might be either reversed or suppressed by correct information or negative expectation, respectively; that expectation of analgesia is a factor in perceived analgesia; that instructions that induce a certain expectation of analgesia induce greater analgesia than those that induce an uncertain expectation; and that negative expectations of analgesia associated with an analgesic intervention might offset some of the effectiveness of that intervention. If pain is indeed a factor in the ergogenic effect of caffeine, all of these findings are of interest to researchers investigating the phenomenon.

Beyond these findings, evidence from over 50 years of scientific research indicates that when a person receives any of a number of biomedical interventions, ranging from tablet to surgery, the brain might play a role in modulating the effectiveness of that intervention. ${ }^{14,40-42}$ Whilst this fact is recognized by sports scientists, and is accounted for in our use of the placebo-controlled experimental design, such psychologic factors are rarely considered legitimate variables in experimental research. This is despite the growing experimental evidence for placebo effects on sports performance. Whilst this literature is reviewed elsewhere, ${ }^{43}$ it suffices to state that placebo effects on sports performance resulting from the belief that an ergogenic substance had been ingested have been reported in 12 well-controlled studies..$^{5-7,39,43-50}$ Most of these effects were in the range of $1 \%-5 \%$. In three studies, nocebo (or negative placebo) effects were observed as the result of subjects either being given negative information about an intervention, ${ }^{44}$ having previous negative experience with caffeine, ${ }^{5}$ or for reasons that were not entirely clear. ${ }^{12}$ Many of the positive effects were similar in magnitude to the effects of the substance that the placebo mimicked. In the few studies in which the biologically active substance was administered alongside the placebo, as is the case in the traditional placebo-controlled study, results were arguably not as easily interpretable as those in which placebos only were administered. This suggests that the interaction of belief and biology in real time, or that order effects in which subjects were able to detect the presence or otherwise of the active substance, might have created tension between information (eg, "Today you have been given a placebo") and perception (eg, 'I'm sure this feels like I have been given caffeine"). This finding in itself warrants further investigation. Whilst knowledge that placebos might be powerful in the absence of the biologically active substance they mimic, such an application is rare in the real world which scientific research aims to inform.

\section{Modifying research designs to understand caffeine effects better}

Burke, in reviewing the caffeine and performance literature, argued strongly for more ecologically valid investigations 
of the phenomenon. ${ }^{2}$ It is fair to argue that, in order to understand any intervention fully, it is necessary to determine how it operates in the real world, outside of the laboratory. However, there are many aspects of the ergogenic effect of caffeine that can be examined in the laboratory, one of which is the contribution or otherwise of psychologic factors. The degree to which the brain is capable of modulating the biologic effect of an intervention, be that modulation trivial, as is likely the case with a strong poison, or substantial, as has been suggested is the case with several complementary medicine treatments, is still little explored in sports performance. Biomedical research is increasingly utilizing sophisticated and elegant designs to investigate this area, and in doing so, is providing a firm biologic basis for what were previously perceived as purely psychologic phenomena. The differences between the laboratory and the field and between research and competition are critical factors. Arguably as important, however, are differences between the subjects' certainty of having received an intervention or of not having done so, or between their lack of faith in an intervention or extreme confidence in the same intervention. Burke also highlighted the interindividual variability in response to caffeine ingestion in performance research. ${ }^{2}$ Much of this variability is likely the result of factors such as habituation, diet, training status, and available physiologic resources. The research described above suggests that, if pain reduction is indeed a mechanism of the ergogenic effects of caffeine, several psychosocial factors might also modulate the ergogenic effects of caffeine. It has been suggested that some athletes are nonresponsive to caffeine. It is, however, possible that if caffeine responses are context-dependent, an athlete who would not respond to caffeine in one context, for example, with no explicit expectation of effect, might respond in another context when given an explicit expectation or when exposed to a conditioning stimulus with false-positive performance outcome feedback. These factors are largely related to the subject's expectations, or conditioned responses, to the caffeine intervention, and these themselves are dependent on either previous experience or currently available information. Therefore, to understand better the effects of caffeine on performance, these psychosocial factors should be treated as variables of interest in caffeine research. Previous experiences of caffeine could be assessed, and in doing so, several aspects of the caffeine experience, including whether the previous intervention had a positive or negative effect on performance, or whether it was associated with any side effects, such as nausea or insomnia, could be factored in. It is also useful to evaluate the subjects' expectations of caffeine. As was demonstrated by
Beedie et al, ${ }^{5}$ expectations of a negative effect can result in a substantial nocebo effect on performance, even in the absence of caffeine. In this respect it is also useful to evaluate whether a subject believes they were given caffeine or placebo in any one trial. Several recent studies have demonstrated that subjects who believed themselves to be in placebo control or no-treatment conditions performed below baseline, suggesting a potentially powerful nocebo effect. Such an effect is perhaps associated with the hope/anticipation of a positive intervention being replaced by the disappointment/anxiety of no intervention. It is important to distinguish between controlling for current use of, or habituation to, caffeine and for previous use. Whilst the physiologic effects of caffeine might be extinct after a few days, conditioned responses, expectations, or both, might persist for several years or even indefinitely.

The suggestion that caffeine's mechanisms might relate in part to psychologic factors is intuitively appealing, especially when considering the multiple feedback loops between brain and body during performance. However, to date, little research in sport has examined, or even considered, such biologic and psychologic interactions in this context. This is not surprising, given the complex links between perception of effort and pain and the biologic reality at that exact point in time. However, if sports scientists are to understand fully the mechanisms of interventions, such questions must, at the very least, be asked, if not immediately or easily answered. A substantial body of biomedical research has demonstrated that brain and body interact in response to the administration of a substance about which the subject has some expectation, be that expectation positive or negative. This is of course the case when an athlete uses caffeine, ie, the athlete has some expectation of an effect. Given the failure to definitively support several longstanding theories of caffeine's ergogenic effects described by Tarnopolsky above, ${ }^{4}$ and the increasing database of studies attesting to the potential psychologic contribution to the action of ergogenic aids in sports performance, ${ }^{42}$ it is perhaps timely to investigate the pain hypothesis, and consider psychosocial variables that might modulate the pain response, in future caffeine and performance research.

\section{Summary}

The ergogenic effects of caffeine have been observed in a number of sports. These effects are more evident in endurance sports or in short-duration, sustained-effort sports. In these sports, athletes would be expected to experience high levels of pain with little relief. There is, however, wide interindividual 
variability in the ergogenic response to caffeine. Placebo effects of caffeine have been observed on sports performance. Given that pain is a limiting factor on performance, that caffeine has well documented hypoalgesic effects, and that pain is highly placebo responsive, pain reduction might be a mechanism in both biologic and placebo caffeine responses in sport. This idea is supported by findings in medicine which suggest that biologic and placebo effects of analgesic drugs share common mechanisms. Researchers should further explore the pain hypothesis and, whilst doing so, consider psychosocial variables that might modulate the pain response in future caffeine and performance research.

\section{Disclosure}

The authors report no conflicts of interest in this work.

\section{References}

1. Graham TE, Battram DS, Dela F, El-Sohemy A, Thong FSL. Does caffeine alter muscle carbohydrate and fat metabolism during exercise? Appl Physiol Nutr Metab. 2008;33:1311-1318.

2. Burke LM. Caffeine and sport performance. Appl Physiol Nutr Metab. 2008;33:1319-1334.

3. Bridge CA, Jones MA. The effect of caffeine ingestion on $8 \mathrm{~km}$ run performance in a field setting. J Sports Sci. 2006;24:433-439.

4. Tarnopolsky MA. Effect of caffeine on the neuromuscular system - potential as an ergogenic aid. Appl Physiol Nutr Metab. 2008; 33:1284-1289.

5. Beedie CJ, Stuart EM, Coleman DA, et al. Placebo effect of caffeine in cycling performance. Med Sci Sports Exerc. 2006;38:2159-2164.

6. Pollo A, Carlino E, Benedetti F. The top-down influence of ergogenic placebos on muscle work and fatigue. Eur J Neurosci. 2008;28:379-388.

7. Foad AJ, Beedie CJ, Coleman DA. Pharmacological and psychological effects of caffeine ingestion in $40 \mathrm{~km}$ cycling performance. Med Sci Sports Exerc. 2008;40(Pt 1):158-165.

8. Kirsch I, Weixel LJ. Double-blind versus deceptive administration of a placebo. Behav Neurosci. 1988;102(Pt 22):319-323.

9. Lotshaw SC, Bradley JR, Brooks LR. Illustrating caffeine's pharmacological and expectancy placebo design. J Drug Educ. 1996;26:13-24.

10. Kirsch I, Rosadino MJ. Do double-blind studies with informed consent yield externally valid results? An empirical test. Psychopharmacology. 1993;110:437-442.

11. Beedie CJ. The placebo effect in competitive sport: Qualitative data. J Sport Sci Med. 2007;6:21-28.

12. Gliottoni RC, Meyers JR, Arngrímsson SA, Broglio SP, Motl RW. Effect of caffeine on quadriceps muscle pain during acute cycling exercise in low versus high caffeine consumers. Int J Sport Nutr Exerc Metab. 2009;19:150-161.

13. Marcora SM, Staiano W. The limit to exercise tolerance in humans: Mind over muscle? Eur J Appl Physiol. Mar 11, 2010. [Epub ahead of print].

14. Benedetti F. Placebo Effects: Understanding the Mechanisms in Health and Disease. Oxford, UK: Oxford University Press; 2009.

15. Graham TE, Spriet LL. Metabolic, catecholamine, and exercise performance responses to various doses of caffeine. J Appl Physiol. 1995;78:867-874.

16. Keogh E, Witt, G. Hypoalgesic effect of caffeine in normotensive men and women. Psychophysiology. 2001;38:886-895.

17. Gliottoni RC, Motl RW. Effect of caffeine on leg muscle pain during intense cycling exercise: Possible role of anxiety sensitivity. Int J Sport Nutr Exerc Metab. 2008;18:103-115.
18. Motl RW, O’Connor PJ, Dishman RK. Effect of caffeine on perceptions of leg muscle pain during moderate intensity cycling exercise. J Pain. 2003;4:316-321.

19. Doherty M, Smith PM. Effects of caffeine ingestion on rating of perceived exertion during and after exercise: A meta-analysis. Scand J Med Sci Sports. 2005;15:69-78.

20. Cook DB. Physical activity and pain. In: Acevedo EO, Ekkekakis P, editors. Psychobiology of Physical Activity. Champaign, IL: Human Kinetics; 2006.

21. Cook DB, O'Connor PJ, Eubanks SA, Smith JC, Lee M. Naturally occurring muscle pain during exercise: Assessment and experimental evidence. Med Sci Sports Exerc. 1997;29:999-1012.

22. O'Connor PJ, Motl RW, Broglio SP, Ely MR. Dose-dependent effect of caffeine on reducing leg muscle pain during cycling exercise is unrelated to systolic blood pressure. Pain. 2004:109:291-298.

23. Maridakis V, O'Connor PJ, Dudley GA, McCully KK. Caffeine attenuates delayed-onset muscle pain and force loss following eccentric exercise. J Pain. 2007;8:237-243.

24. Montgomery GH, Kirsch I. Classical conditioning and the placebo effect. Pain. 1997;72:107-113.

25. Levine JD, Gordon NC, Smith R, Feilds HL. Analgesic responses to morphine and placebo in individuals with post-operative pain. Pain. 1981;10:379-389.

26. Levine JD, Gordon NC. Influence of the method of drug administration on analgesic response. Nature. 1984;312:755-756.

27. Amanzio M, Pollo A, Maggi G, Benedetti F. Response variability to analgesics: A role for non-specific activation of endogenous opioids. Pain. 2001;90:205-215.

28. Benedetti F, Maggi G, Lopiano L, et al. Open versus hidden medical treatments: The patient's knowledge about a therapy affects the therapy outcome. Prevent Treat. 2003. Available from http://psycnet.apa.org/ journals/pre/6/1/1a/. Accessed May 18, 2010.

29. Colloca L, Lopiano L, Lanotte M, Benedetti F. Overt versus covert treatment for pain, anxiety, and Parkinson's disease. Lancet Neurol. 2004;3:679-684.

30. Pollo A, Amanzio M, Arslanian A, Casadio C, Maggi G, Benedetti F. Response expectancies in placebo analgesia and their clinical relevance. Pain. 2001;93:77-84.

31. Vase L, Riley JL, Price DD. A comparison of placebo effects in clinical analgesic trials versus studies of placebo analgesia. Pain. 2002;99: 443-452.

32. Benedetti F, Amanzio M, Casadio C, Oliaro A, Maggi G. Blockade of nocebo hyperalgesia by the cholecystokinin antagonist proglumide. Pain. 1997;71:135-140.

33. Benedetti F, Arduino C, Costa S, et al. Loss of expectation-related mechanisms in Alzheimer's disease makes analgesic therapies less effective. Pain. 2006;121:133-144.

34. Levine, JD, Gordon NC, Fields HL. The mechanism of placebo analgesia. Lancet. 1978;312:654-657.

35. Benedetti F. The opposite effects of the opiate antagonist naloxone and the cholecystokinin antagonist proglumide on placebo analgesia. Pain. 1996;64:535-543.

36. Petrovic, P, Kalso E, Petersson KM, Ingvar M. Placebo and opioid analgesia - imaging a shared neuronal network. Science. 2002;295: $1737-1740$.

37. WagerTD, Rilling JK, Smith EE, et al. Placebo-induced changes in fMRI in the anticipation and experience of pain. Science. 2004;303:1162-1166.

38. Scott DJ, Stohler CS, Egnatuk CM, Wang H, Koepp RA, Zubietta JK. Placebo and nocebo effects are defined by opposite and opioid and dopaminergic responses. Arch Gen Psychiatry. 2007;65:220-231.

39. Benedetti F, Pollo A, Colloca L. Opioid-mediated placebo responses boost pain endurance and physical performance: Is it doping in sport competitions? J Neurosci. 2007;27:11934-11939.

40. Evans D. Placebo: The Belief Effect. London, UK: Harper Collins; 2003.

41. Kirsch I. The Emperor's New Drugs: Exploding the Antidepressant Myth. London, UK: Bodley Head; 2009. 
42. Beedie CJ, Foad AJ. The placebo effect in sport. A brief review. Sports Med. 2009;39:313-329.

43. Ariel G, Saville W. Anabolic steroids: The physiological effects of placebos. Med Sci Sports Exerc. 1972;4:124-126.

44. Beedie CJ, Coleman DA, Foad AJ. Positive and negative placebo effects resulting from the deceptive administration of an ergogenic aid. Int J Sport Nutr Exerc Metab. 2007;17:259-269.

45. Clark VR, Hopkins WG, Hawley JA, et al. Placebo effect of carbohydrate feeding during a 40-km cycling time trial. Med Sci Sports Exerc. 2000; 32:1642-1647.

46. Foster C, Felker H, Porcari JP, et al. The placebo effect on exercise performance. Med Sci Sports Exerc. 2004;36 Suppl 5:Abstr S171.

47. Kalasountas V, Reed J, Fitzpatrick J. The effect of placebo induced changes in expectancies on maximal force production in college students. J Appl Sport Psychol. 2007;19(Pt 1):116-124.
48. Maganaris CN, Collins D, Sharp M. Expectancy effects and strength training: Do steroids make a difference? Sport Psychologist. 2000; 14(Pt 3):272-278

49. McClung M, Collins D. "Because I know it will!": Placebo effects of an ergogenic aid on athletic performance. J Sport Exerc Psychol. 2007;29(Pt 3):382-394.

50. Porcari J, Foster C. Mind over body: ACE fitness matters 2006. Available from: http://www.acefitness.org/getfit/PlaceboStudy2006.pdf. Accessed Dec 5, 2006.

\section{Publish your work in this journal}

Open Access Journal of Sports Medicine is an international, peer-reviewed, open access journal publishing original research, reports, reviews and commentaries on all areas of sports medicine. The manuscript management system is completely online and includes a very quick and fair peer-review system.
Visit http://www.dovepress.com/testimonials.php to read real quotes from published authors. 